\title{
EULER CHARACTERISTICS OF ARITHMETIC GROUPS
}

\author{
I. Horozov
}

\begin{abstract}
We have developed a general method for computing the homological Euler characteristic of finite index subgroups $\Gamma$ of $G L_{m}\left(\mathcal{O}_{K}\right)$ where $\mathcal{O}_{K}$ is the ring of integers in a number field $K$. With this method we find, that for large, explicitly computed dimensions $m$, the homological Euler characteristic of finite index subgroups of $G L_{m}\left(\mathcal{O}_{K}\right)$ vanishes. For other cases, some of them very important for spaces of motivic multiple polylogarithms at $n$-th root of unity, we compute non-zero homological Euler characteristic. Finally, our method allows us to obtain a formula for the Dedekind zeta function at -1 in terms of the ideal class set and the multiplicative group of quadratic extensions of the base ring.
\end{abstract}

\section{Introduction}

The homological Euler characteristic of a group $\Gamma$ with coefficients in a representation $V$ is defined by

$$
\chi_{h}(\Gamma, V)=\sum_{i}(-1)^{i} \operatorname{dim} H^{i}(\Gamma, V)
$$

(see [S], [B1]). The main result of our paper is a general method that allows us to calculate $\chi_{h}(\Gamma, V)$. Toward the end of the introduction we describe briefly this method. Before that we list the most important results of the paper which demonstrate the scope of the method. Our first result is about vanishing of homological Euler characteristics.

Theorem 0.1. Let $\Gamma$ be a subgroup of $G L_{m}(K)$, commensurable to $G L_{m}\left(\mathcal{O}_{K}\right)$, where $\mathcal{O}_{K}$ is the ring of integers in a number field $K$. Let $V$ be a finite dimensional representation of $\Gamma$. Then $\chi_{h}(\Gamma, V)=0$ if

(a) $K=\mathbb{Q}$ if $m>10$

(b) $K=\mathbb{Q}(\sqrt{-d})$, where $-d$ is the discriminant,

if $d=4$ and $m>4$

if $d=3$ and $m>6$

for the other d's and $m>2$

(c) for the remaining number fields $K$.

We obtain a similar result for $S L_{m}$ in place of $G L_{m}$.

Received March 23, 2004. 
Theorem 0.2. Let $\Gamma$ be a subgroup of $S L_{m}(K)$, commensurable to $S L_{m}\left(\mathcal{O}_{K}\right)$, where $m \geq 2$ and $\mathcal{O}_{K}$ is the ring of integers in a number field $K$. Let $V$ be a finite dimensional representation of $\Gamma$. Then $\chi_{h}(\Gamma, V)=0$ if

(a) $K=\mathbb{Q}$ if $m>10$

(b) $K=\mathbb{Q}(\sqrt{-d})$, if $d=4$ and $m>3$, if $d=3$ and $m>5$, for the other d's,

(c) $K$ is a totally real field and $m>2$,

(d) for the remaining number fields $K$.

For $S p_{m}$ we have similar result.

Theorem 0.3. Let $\Gamma$ be a subgroup of $S p_{m}(K)$, commensurable to $S p_{m}\left(\mathcal{O}_{K}\right)$, $\mathcal{O}_{K}$ is the ring of integers in a number field $K$. Let $V$ be a finite dimensional representation of $\Gamma$. Then $\chi_{h}(\Gamma, V)=0$ if

(a) $K=\mathbb{Q}$ (i) if $m>1$,

(b) $K=\mathbb{Q}\left(\xi_{3}\right)$ if $m>2$,

(c) $K$ is different from the above two and not a totally real field.

We also compute the homological Euler characteristic of the arithmetic subgroups $\Gamma_{1}(3, N)$ and $\Gamma_{1}(4, N)$ of $G L_{3}(\mathbb{Z})$ and $G L_{4}(\mathbb{Z})$, respectively, where $\Gamma_{1}(m, N)$ is the subgroup of $G L_{m}(\mathbb{Z})$ that fixes the vector $[0, \ldots, 0,1] \bmod N$.

Theorem 0.4. The homological Euler characteristic of $\Gamma_{1}(3, N)$ and of $\Gamma_{1}(4, N)$ for $N$ not divisible by 2 and 3 is given by

$$
\begin{gathered}
\chi_{h}\left(\Gamma_{1}(3, N), \mathbb{Q}\right)=-\frac{1}{12} \varphi_{2}(N)+\frac{1}{2} \varphi(N), \\
\chi_{h}\left(\Gamma_{1}(4, N), \mathbb{Q}\right)=\varphi(N),
\end{gathered}
$$

where $\varphi(N)$ is the Euler $\varphi$-function, and $\varphi_{2}(N)$ is the multiplicative arithmetic function generated by $\varphi_{2}\left(p^{a}\right)=p^{2 a}\left(1-\frac{1}{p^{2}}\right)$ for $a \geq 1$.

Using our method we also compute $\chi_{h}\left(G L_{m}(\mathbb{Z}), S^{n} V_{m}\right)$ for $m=3$ and $m=4$, where $S^{n} V_{m}$ is the $n$-th symmetric power of the standard $m$ dimensional representation of $G L_{m}(\mathbb{Z})$. The computation of $\chi_{h}\left(G L_{3}(\mathbb{Z}), S^{n} V_{3}\right.$ ) (see theorems 3.1 and 3.2$)$ agrees with the computation of the $H^{i}\left(G L_{3}(\mathbb{Z}), S^{n} V_{3}\right)$ in [G1], which was used for computation of dimensions of spaces of certain motivic multiple polylogarithms. Also the homological Euler characteristic of $\Gamma_{1}(3, N)$ with 
trivial coefficients when $N$ is an odd prime, greater that 3 , agrees with the computation of $H_{i n f}^{i}\left(\Gamma_{1}(3, N), \mathbb{Q}\right)$ in the corrected version of [G1]. We compute the homological Euler characteristic of $G L_{2}(\mathbb{Z}[i])$ and $G L_{2}\left(\mathbb{Z}\left[\xi_{3}\right]\right)$ with coefficients in the symmetric powers of the standard representations. Also we compute the homological Euler characteristic of $\Gamma_{1}(2, \mathfrak{a})$ for an ideal $\mathfrak{a}$ in $Z[i]$ and $\mathbb{Z}\left[\xi_{3}\right]$, respectively.

Theorem 0.5. (a) If $1+i$ does not divide $\mathfrak{a}$ the homological Euler characteristic of $\Gamma_{1}(2, \mathfrak{a}) \subset G L_{2}(\mathbb{Z}[i])$ is given by

$$
\chi_{h}\left(\Gamma_{1}(2, \mathfrak{a}), \mathbb{Q}\right)=\frac{1}{2} \varphi_{\mathbb{Z}[i]}(\mathfrak{a}),
$$

where $\varphi_{\mathbb{Z}[i]}(\mathfrak{a})$ is the multiplicative function defined on the ideals of $\mathbb{Z}[i]$, generated by

$$
\varphi_{\mathbb{Z}[i]}\left(\mathfrak{p}^{n}\right)=N_{\mathbb{Q}(i) / \mathbb{Q}}(\mathfrak{p})^{n}\left(1-\frac{1}{N_{\mathbb{Q}(i) / \mathbb{Q}}(\mathfrak{p})}\right) .
$$

(b) If $1+\xi_{6}$ does not divide $\mathfrak{a}$ the homological Euler characteristic of $\Gamma_{1}(2, \mathfrak{a}) \subset$ $G L_{2}\left(\mathbb{Z}\left[\xi_{3}\right]\right)$ is given by

$$
\chi_{h}\left(\Gamma_{1}(2, \mathfrak{a}), \mathbb{Q}\right)=\frac{1}{3} \varphi_{\mathbb{Z}\left[\xi_{3}\right]}(\mathfrak{a}),
$$

where $\varphi_{\mathbb{Z}\left[\xi_{3}\right]}(\mathfrak{a})$ is the multiplicative function defined on the ideals of $\mathbb{Z}\left[\xi_{3}\right]$, generated by

$$
\varphi_{\mathbb{Z}\left[\xi_{3}\right]}\left(\mathfrak{p}^{n}\right)=N_{\mathbb{Q}\left(\xi_{3}\right) / \mathbb{Q}}(\mathfrak{p})^{n}\left(1-\frac{1}{N_{\mathbb{Q}\left(\xi_{3}\right) / \mathbb{Q}}(\mathfrak{p})}\right) .
$$

In general the method works for any arithmetic subgroup of $G L_{m}\left(\mathcal{O}_{K}\right)$, where $\mathcal{O}_{K}$ is the ring of integers in a number field $K$.

Our approach is the following: we generalize a result of $\mathrm{K}$. Brown [B2] that relates the torsion elements in the group up to conjugation to the homological Euler characteristic of the group. Namely,

$$
\chi_{h}(\Gamma, V)=\sum_{T} \chi(C(T)) \operatorname{Tr}\left(T^{-1} \mid V\right)
$$

where the sum is over all torsion elements $T$ of $\Gamma$ up to conjugation and $C(T)$ is the centralizer of $T$ in $\Gamma$. Let us recall the definition of orbifold Euler characteristic of $\Gamma$, denoted by $\chi(\Gamma)$, which we simply call Euler characteristic. If $\Gamma$ is a torsion free group then $\chi(\Gamma)=\chi_{h}(\Gamma)$. If $\Gamma$ has torsion consider a finite index torsion free subgroup $\Gamma^{\prime}$. Then $\chi(\Gamma)$ is defined by

$$
\chi(\Gamma)=\left[\Gamma: \Gamma^{\prime}\right]^{-1} \chi\left(\Gamma^{\prime}\right) .
$$

Arithmetic groups do have a finite index torsion free subgroup. So for them the Euler characteristic is defined. The main properties of the Euler characteristic that we are going to use are:

$$
\chi(G)=\frac{1}{|G|} \text { for a finite group } \mathrm{G},
$$


Given an exact sequence

$$
0 \rightarrow \Gamma_{1} \rightarrow \Gamma \rightarrow \Gamma_{2} \rightarrow 0
$$

we have

$$
\chi(\Gamma)=\chi\left(\Gamma_{1}\right) \chi\left(\Gamma_{2}\right)
$$

and

$$
\chi\left(S L_{m}\left(\mathcal{O}_{K}\right)\right)=\zeta_{K}(-1) \ldots \zeta_{K}(1-m) .
$$

The first two properties can be found in K. Brown's book [B1]. And the last one is a difficult result due to Harder $[\mathrm{H}]$.

In order to use the Brown's formula we need to know the torsion elements in the group. We develop a method for finding the torsion elements in $G L_{m}\left(\mathcal{O}_{K}\right)$, and consider another form of the above formula that requires very few of the torsion elements ( in general they can be quite a large number). This method involves linear algebra over number rings. More precisely is describes a normal form of matrices over number ring. First we deal with matrices with irreducible characteristic polynomial which lead to a relation to ideal classes (proposition 1.3). Then we examine matrices with reducible characteristic polynomials which leads to resultants (lemma 2.2 and proposition 2.3). Then we examine the relation between the torsion elements in $G L_{m}\left(\mathcal{O}_{K}\right)$ and the torsion elements in an arithmetic subgroup $\Gamma$ of $G L_{m}\left(\mathcal{O}_{K}\right)$. That gives us the homological Euler characteristic of $\Gamma$ with coefficients in a representation.

We obtain another interesting application of our method for computation of the values of the Dedekind zeta functions at -1 . Now we are going to explain how this is related to our method. First, the Dedekind zeta function at -1 vanishes for number fields which are not totally real. For totally real number fields $K$ we consider the arithmetic group $S L_{2}\left(\mathcal{O}_{K}\right)$. By a result of Harder $[\mathrm{H}]$ we have that the orbifold Euler characteristic of $S L_{2}\left(\mathcal{O}_{K}\right)$ is the Dedekind zeta function at $-1 \zeta_{K}(-1)$. Also the homological Euler characteristic is always an integer. Let us denote the homological Euler characteristic by an integer $N$, i.e

$$
N=\chi_{h}\left(S L_{2}\left(\mathcal{O}_{K}\right)\right) \text {. }
$$

We also introduce the following notation. Let

$$
\operatorname{Cl}\left(\mathcal{O}_{K}[\xi] / \mathcal{O}_{K}\right)
$$

be the set of ideal classes in $\mathcal{O}_{K}[\xi]$ which are free as $\mathcal{O}_{K}$-modules. Let also $R_{I} \subset \mathcal{O}_{K}[\xi]$ be isomorphic to the ring of matrices that commute with $A$, where $I$ the ideal corresponding to the matrix $A$ by proposition 1.3.

Theorem 0.6. Let $K$ be a totally real number field. Let $R$ be the ring of integers in $K$. Then the Dedekind zeta function at -1 can be expressed as

$$
\zeta_{K}(-1)=-\frac{1}{4} \sum_{\xi} \sum_{I \in C l\left(\mathcal{O}_{K}[\xi] / \mathcal{O}_{K}\right)} \frac{\# \mathcal{O}_{K}^{\times} / N_{K(\xi) / K}\left(R_{I}^{\times}\right)}{\#\left(R_{I}^{\times}\right)_{\text {tors }}}+\frac{1}{2} N,
$$

where the the first sum is taken over all roots of 1 such that $[K(\xi): K]=2$, the second sum over all ideal classes in $\mathcal{O}_{K}[\xi]$ that are free as $\mathcal{O}_{K}$-modules, the 
ring $R_{I}$ sits between $\mathcal{O}_{K}[\xi]$ and its integral closure $\mathcal{O}_{K(\xi)} . R_{I}$ is as above, and $N=\chi_{h}\left(S L_{2}\left(\mathcal{O}_{K}\right), \mathbb{Q}\right)$.

Note that this formula gives that the denominator of $\zeta_{K}(-1)$ is related to the torsion elements of the quadratic cyclotomic extensions of $K$. This is the same as the Tate constant $w_{2}$ (see $\left.[\mathrm{T}]\right)$.

The organization of the paper is the following. In section 1 we deals with linear algebra over rings of algebraic integers. We give a method for classification of the matrices with integer coefficients for the ones that are diagonalizable over the complex numbers. In section 2 we give a method of computing the centralizer of a matrix which leads to explicit formulas for the homological Euler characteristics of $G L_{m} \mathbb{Z}, G L_{m}(\mathbb{Z}[i]), G L_{m}\left(\mathbb{Z}\left[\xi_{3}\right]\right)$ and $G L_{2}\left(\mathcal{O}_{\mathbb{Q}(\sqrt{-d})}\right)$, see respectively theorems 2.9, 2.10, 2.11 and 2.12. In section 3 we find the homological Euler characteristics of $G L_{m}\left(\mathcal{O}_{K}\right)$ with coefficients the symmetric powers of the standard representation. Also, we find the homological Euler characteristics of arithmetic groups $\Gamma_{1}(m, N)$ and $\Gamma_{1}(m, \mathfrak{a})$. In the end of the section, we examine the groups $S L_{2}\left(\mathcal{O}_{K}\right)$ for totally real number fields $K$ which gives a relation to the Dedekind zeta function of the field. And in last section we prove the generalization of Brown's formula.

\section{Conjugacy classes in $G L_{m}\left(\mathcal{O}_{K}\right)$}

In this section we describe the conjugacy classes of elements in $G L_{m}\left(\mathcal{O}_{K}\right)$, which are semi-simple over the complex numbers, where $\mathcal{O}_{K}$ is the ring of integers in a number field $K$. We approach the description of conjugacy classes in the following way. We examine matrices in $G L_{m}\left(\mathcal{O}_{K}\right)$ whose characteristic polynomial is irreducible over $K$. They are described by ideal classes of a larger ring. And, finally, we construct an algorithm for matrices with reducible characteristic polynomial that allows to consider instead matrices of smaller dimension. Using this inductive step we can describe completely conjugacy classes of certain type, knowing the conjugacy classes of matrices of smaller dimension.

This subsection deals with the case when the characteristic polynomial of a matrix is reducible over a field $K$. The partition of a matrix into blocks will be done in the following way: Given an $m \times m$ matrix $A$, let $m=m_{1}+\cdots+m_{k}$ be a partition of $n$. Then $A$ can be thought of as a $k \times k$ block-matrix whose $A_{i j}$-entry, $i, j=1, \ldots k$ is a block (and a matrix) of size $m_{i} \times m_{j}$. This will be the type of block-matrices that we consider. Note that the blocks $A_{i i}$ are square matrices.

Definition 1.1. Let $P$ be a finitely generated torsion free module over an integral Noetherian ring $R$ of dimension 1 . Let $K$ be the field of fractions of $R$. Let $A$ be an endomorphism of $P$,

$$
A \in \operatorname{End}_{R}(P)
$$


Then $A$ can be extended to an endomorphism of a finite dimensional vector space $P \otimes_{R} K$. Then the characteristic polynomial of $A$ is defined to be the characteristic polynomial of the induced map in $\operatorname{End}_{K}\left(P \otimes_{R} K\right)$.

Theorem 1.2. Let $R$ be a Dedekind domain. And let $P$ be a finitely generated projective module over $R$. Assume that the field of fractions $K$ has characteristic 0. Let, also, $A$ be an endomorphism of $P$. Then in a suitable basis we have

$$
A=\left[\begin{array}{ccc}
A_{11} & \cdots & A_{1 d} \\
0 & \ddots & \vdots \\
0 & 0 & A_{d d}
\end{array}\right]
$$

where $A_{i j} \in \operatorname{Hom}\left(P_{j}, P_{i}\right)$, with $P=P_{1} \oplus \cdots \oplus P_{d}$, so that $A_{i j}=0$ for $i>j$ and $A_{i i}$ has irreducible over $K$ characteristic polynomial.

We have the following characterization of matrices with an irreducible characteristic polynomial.

Proposition 1.3. Let $f(t)$ be an irreducible monic polynomial. And let $P$ be a projective $\mathcal{O}_{K}$-module. Consider the set of all endomorphisms $\left\{A_{i}\right\}$ of $P$ which have characteristic polynomial $f(t)$ and which are not conjugate to each other via an automorphism of $P$. This set is parameterized by the ideal classes in $\mathcal{O}_{K}[t] /(f(t))$ which are isomorphic to $P$ as $\mathcal{O}_{K}$-modules.

Corollary 1.4. Let $f(t) \in \mathcal{O}_{K}[t]$ be an irreducible monic polynomial. Assume that $\mathcal{O}_{K}[t] /(f(t))$ is a integrally closed. Then the conjugacy classes of matrices in $G L_{m}\left(\mathcal{O}_{K}\right)$ with characteristic polynomial $f(t)$ are in one-to-one correspondence with the elements in

$$
\operatorname{Ker}\left(K_{0}\left(\mathcal{O}_{K}[t] /(f(t))\right) \rightarrow K_{0}\left(\mathcal{O}_{K}\right)\right) .
$$

Using the Theorem 1.2 we obtain the following: If $A$ is an $m \times m$ matrix with coefficients in $\mathcal{O}_{K}$, or more generally, an endomorphism of projective $\mathcal{O}_{K}$-module of rank $m$, having a reducible characteristic polynomial then $A$ is conjugated by an element of $G L_{m}\left(\mathcal{O}_{K}\right)$, or of $A u t(P)$, to a $2 \times 2$-block endomorphism

$$
A=\left[\begin{array}{cc}
A_{11} & A_{12} \\
0 & A_{22}
\end{array}\right] \text {. }
$$

Let $A_{11}$ and $A_{22}$ be automorphisms of projective modules $P_{1}$ and $P_{2}$, with $P_{1} \oplus P_{2}=P$. Now we are going to describe a method that simplifies the block $A_{12}$ and leaves $A_{11}$ and $A_{22}$ unchanged. Conjugate $A$ with an automorphism

$$
B=\left[\begin{array}{cc}
B_{11} & B_{12} \\
0 & B_{22}
\end{array}\right]
$$

of the some block form. that is $B_{11} \in \operatorname{Aut}\left(P_{1}\right), B_{22} \in \operatorname{Aut}\left(P_{2}\right)$ and $B_{12} \in$ $\operatorname{Hom}\left(P_{2}, P_{1}\right)$. We want the conjugation by $P$ to preserve $A_{11}$ and $A_{22}$ that is

$$
\left[\begin{array}{cc}
B_{11} & B_{12} \\
0 & B_{22}
\end{array}\right] \cdot\left[\begin{array}{cc}
A_{11} & A_{12} \\
0 & A_{22}
\end{array}\right]=\left[\begin{array}{cc}
A_{11} & A_{12}^{\prime} \\
0 & A_{22}
\end{array}\right] \cdot\left[\begin{array}{cc}
B_{11} & B_{12} \\
0 & B_{22}
\end{array}\right] .
$$


We do have that $A_{12}$ is changed to $A_{12}^{\prime}$. Then

$$
B_{11} A_{11}=A_{11} B_{11}, B_{22} A_{22}=A_{22} B_{22},
$$

and

$$
A_{12}^{\prime} B_{22}-B_{11} A_{12}=B_{12} A_{22}-A_{11} B_{12} .
$$

Denote by $C\left(A_{i i}\right)$ the centralizer of $A_{i i}$. Then $B_{11} \in C\left(A_{11}\right)$ and $B_{22} \in C\left(A_{22}\right)$. The block $B_{12}$ could be any map in $\operatorname{Hom}_{\mathcal{O}_{K}}\left(P_{2}, P_{1}\right)$. Let

$$
P_{A_{11}, A_{22}}: B_{12} \mapsto B_{12} A_{22}-A_{11} B_{12}
$$

be a map from the space $\operatorname{Hom}_{\mathcal{O}_{K}}\left(P_{2}, P_{1}\right)$ to itself. It is linear. Then the relation

$$
A_{12}^{\prime} B_{22}-B_{11} A_{12}=B_{12} A_{22}-A_{11} B_{12},
$$

between $A_{12}^{\prime}$ and $A_{12}$ can be written as

$$
A_{12}^{\prime} B_{22} \equiv B_{11} A_{12} \bmod \left(\operatorname{Im} P_{A_{11}, A_{22}}\right) .
$$

Lemma 1.5. Let $P_{\text {mod }}=\operatorname{Im}\left(P_{A_{11}, A_{22}}\right)$ where $P_{A_{11}, A_{22}}: B_{12} \mapsto B_{12} A_{22}-$ $A_{11} B_{12}$ Let also $Q_{\text {mod }}=\operatorname{Hom}_{\mathcal{O}_{K}}\left(P_{2}, P_{1}\right) / P_{\text {mod }}$. Then $\operatorname{Hom}_{\mathcal{O}_{K}}\left(P_{2}, P_{1}\right), P_{\text {mod }}$ and $Q_{\text {mod }}$ are $C\left(A_{11}\right) \times C\left(A_{22}\right)$-modules.

Proof. Let $B_{11} \in C\left(A_{11}\right)$ and $B_{22} \in C\left(A_{22}\right)$. Then

$$
\left(B_{11}, B_{22}\right) \cdot P_{A_{11}, A_{22}}\left(B_{12}\right)=P_{A_{11}, A_{22}}\left(B_{11} B_{12} B_{22}^{-1}\right) .
$$

Obviously, $\operatorname{Hom}_{\mathcal{O}_{K}}\left(P_{2}, P_{1}\right)$ is a $C\left(A_{11}\right) \times C\left(A_{22}\right)$-module. Thus, the quotient, as abelian group, $Q_{\text {mod }}=\operatorname{Hom}_{\mathcal{O}_{K}}\left(P_{2}, P_{1}\right) / P_{\text {mod }}$ has the structure of a $C\left(A_{11}\right) \times$ $C\left(A_{22}\right)$-module.

Proposition 1.6. Let $A_{11} \in \operatorname{Aut}\left(P_{1}\right)$ and $A_{22} \in \operatorname{Aut}\left(P_{2}\right)$. Suppose $A_{11}$ and $A_{22}$ have no common eigenvalues. Then the matrices

$$
\left[\begin{array}{cc}
A_{11} & A_{12} \\
0 & A_{22}
\end{array}\right] \text { and }\left[\begin{array}{cc}
A_{11} & A_{12}^{\prime} \\
0 & A_{22}
\end{array}\right]
$$

are conjugate to each other if and only if the projection of $A_{12}$ and $A_{12}^{\prime}$ onto the finite set $C\left(A_{11}\right) \times C\left(A_{22}\right) \backslash Q_{\text {mod }}$ coincide.

Lemma 1.7. If $\prod_{i}\left(t-\alpha_{i}\right)$ and $\prod_{j}\left(t-\beta_{j}\right)$ are the characteristic polynomials of $A_{11} \in G L_{m_{1}} \bar{K}$ and $A_{22} \in G L_{m_{2}} \bar{K}$ then the characteristic polynomial of

$$
P_{A_{11}, A_{22}}: X \mapsto X A_{22}-A_{11} X
$$

is $\prod_{i, j}\left(t-\beta_{j}+\alpha_{i}\right)$. In particular, If $A_{11}$ and $A_{22}$ have no common eigenvalue then the map $P_{A_{11}, A_{22}}$ is non-singular, and $Q_{\text {mod }}$ is finite, namely the norm of the resultant. 


\section{Centralizers and homological Euler characteristics}

Lemma 2.1. Let $A_{11}$ and $A_{22}$ be square matrices with coefficients in $\mathcal{O}_{K}$. Suppose that they have no common eigenvalues. And let

$$
C=\left[\begin{array}{ll}
C_{11} & C_{12} \\
C_{21} & C_{22}
\end{array}\right] \text { commutes with } A=\left[\begin{array}{cc}
A_{11} & A_{12} \\
0 & A_{22}
\end{array}\right]
$$

Then the admissible matrices $C$ are determined by the following properties:

$$
\begin{gathered}
C_{21}=0, \\
C_{11} \in C\left(A_{11}\right), \\
C_{22} \in C\left(A_{22}\right), \\
C_{11} A_{12} C_{22}^{-1} \equiv A_{12} \bmod \operatorname{Im} P_{A_{11} A_{22}} .
\end{gathered}
$$

Also, the matrix $C_{12}$ is uniquely determined by $C_{11}$ and $C_{22}$, and it is given by

$$
C_{12}=P_{A_{11} A_{22}}^{-1}\left(A_{12} C_{22}-C_{11} A_{12}\right) \text {. }
$$

In particular,

$$
C\left(\left[\begin{array}{cc}
A_{11} & 0 \\
0 & A_{22}
\end{array}\right]\right)=C\left(A_{11}\right) \times C\left(A_{22}\right),
$$

where $C(A)$ denotes the centralizer of $A$.

Lemma 2.2. Let $A_{11}$ and $A_{22}$ be invertible matrices with coefficients in $\mathcal{O}_{K}$, or automorphisms of projective modules having no common eigenvalues. Let $f_{1}$ and $f_{2}$ be the characteristic polynomials if $A_{11}$ and $A_{22}$, respectively. Then

$$
\sum \chi(C(A))=\left|N_{K / \mathbb{Q}}\left(R\left(f_{1}, f_{2}\right)\right)\right| \chi\left(C\left(A_{11}\right)\right) \chi\left(C\left(A_{22}\right)\right),
$$

where the sum is taken over all non-conjugate torsion elements

$$
A=\left[\begin{array}{cc}
A_{11} & A_{12} \\
0 & A_{22}
\end{array}\right]
$$

with fixed $A_{11}$ and $A_{22}$, and $R\left(f_{1}, f_{2}\right)$ is the resultant of the two polynomials.

Proof. By Proposition 1.6, taking the sum over all non-conjugate matrices with fixed $A_{11}$ and $A_{22}$ is the same as varying $A_{12}$ through representatives of

$$
C\left(A_{11}\right) \times C\left(A_{22}\right) \backslash Q_{\text {mod }} .
$$

For a fixed $A_{12}$ we have that the group $C(A)$ is a finite index subgroup of $C\left(A_{11}\right) \times C\left(A_{22}\right)$, and the index is equal to the number of elements in the $C\left(A_{11}\right) \times C\left(A_{22}\right)$-orbit of $A_{12}$ in $Q_{\text {mod }}$. Thus,

$$
\chi(C(A))=\# \mid \text { orbit of } A_{12} \mid \cdot \chi\left(C\left(A_{11}\right)\right) \chi\left(C\left(A_{22}\right)\right)
$$

Summing over all orbits, we obtain

$$
\sum \chi(C(A))=\#\left|Q_{\text {mod }}\right| \cdot \chi\left(C\left(A_{11}\right)\right) \chi\left(C\left(A_{22}\right)\right) .
$$


On the other hand, by lemma 1.7

$$
\#\left|Q_{\text {mod }}\right|=\left|N_{K / \mathbb{Q}}\left(\operatorname{det}\left(P_{A_{11} A_{22}}\right)\right)\right|=\left|N_{K / \mathbb{Q}}\left(R\left(f_{1}, f_{2}\right)\right)\right| .
$$

We are going to define a resultant of $k$ polynomials $f_{1}, \ldots, f_{k}, k \geq 2$ by

$$
R\left(f_{1}, \ldots, f_{k}\right)=\prod_{i<j} R\left(f_{i}, f_{j}\right) .
$$

Using the previous lemma as an induction step, we obtain the following proposition

Proposition 2.3. Let $A_{11}, A_{22}, \cdots, A_{k k}$ be automorphisms of projective modules over $\mathcal{O}_{K}$ such that $A_{i i}$ and $A_{j j}$ have no common eigenvalues for $i \neq j$. Let $f_{i}$ be the characteristic polynomial of $A_{i i}$. Then

$$
\sum \chi(C(A))=\left|N_{K / \mathbb{Q}}\left(R\left(f_{1}, \ldots, f_{k}\right)\right)\right| \cdot \chi\left(C\left(A_{11}\right)\right) \ldots \chi\left(C\left(A_{k k}\right)\right)
$$

where the sum is taken over all non-conjugate torsion elements $A$ is a blockdiagonal form such that the blocks on the diagonal are $A_{11}, \ldots, A_{k k}$, and the blocks under the diagonal are zero, and $R\left(f_{1}, \ldots, f_{k}\right)$ is the resultant of $f_{1}, \ldots, f_{k}$ defined above.

Note that all $A$ 's in the above sum have the same characteristic polynomial; namely $f_{1} \cdots f_{k}$.

We need e few more lemmas on the size of the centralizer.

Lemma 2.4. Let $A$ and $B$ be matrices in $G L_{m} \mathcal{O}_{K}$, or $\operatorname{Aut}(P)$ which are conjugate as elements of $G L_{m} K$ the $C(A)$ and $C(B)$ are commensurable.

Proof. The group $C(A)$ is an arithmetic subgroup of $C_{G L_{m} K}(A)$, which is conjugate to $C_{G L_{m} K}(B)$. Therefore $C_{G L_{m} \mathcal{O}_{K}}(A)$ and $C_{G L_{m} \mathcal{O}_{K}}(B)$ are commeasurable.

Lemma 2.5. Let $T_{n} \in G L_{m}\left(\mathcal{O}_{K}\right)$ be an $n$-torsion matrix with with irreducible characteristic polynomial. Then the centralizer $C\left(T_{n}\right)$ contains $\mathcal{O}_{K}\left[\xi_{n}\right]^{\times}$, and is commensurable to it where $\xi_{n}=e^{2 \pi i / n}$.

Proof. The matrices in $M a t_{m, m} K$ commuting with $T_{n}$ are precisely

$$
K\left[T_{n}\right] \cong K\left(\xi_{n}\right)
$$

because this is the maximal abelian sub-Lie algebra commuting with $T_{n}$; it is of dimension $m$. Let the intersection of $K\left[T_{n}\right]$ with $M a t_{m, m} \mathcal{O}_{K}$ be

$$
R \subset \mathcal{O}_{K\left(\xi_{n}\right)},
$$

where $R$ and $\mathcal{O}_{K\left(\xi_{n}\right)}$ are commensurable by the previous lemma. In this intersection $R$ the invertible elements are

$$
C\left(T_{n}\right) \cong R^{\times} \subset \mathcal{O}_{K\left[\xi_{n}\right]}^{\times} .
$$


Lemma 2.6. Let $T_{n} \in G L_{m} \mathbb{Z}$ be an $n$-torsion matrix with irreducible characteristic polynomial. Let $T$ be a $k \times k$-block matrix with blocks on the diagonal $T_{n}$ and the rest of the blocks being zero. Let $R$ be the ring of endomorphisms that commute with $T_{n}$. Then $C(T) \cong G L_{k} R$.

Proof. Then the matrices commuting with $T$ are

$$
\mathbb{Q}\left[T_{n}\right] \otimes \operatorname{Mat}_{k, k} K \cong \operatorname{Mat}_{k, k}\left(K\left(\xi_{n}\right)\right) .
$$

Among them the ones with integer coefficients are

$$
C_{M_{t_{k m} \mathbb{Z}}}(T)=C_{\text {Mat }_{k m} \mathbb{Z}}\left(T_{n} \otimes I_{k}\right)=C_{\text {Mat }_{k} \mathbb{Z}}\left(T_{n}\right) \otimes \operatorname{Mat}_{k, k} \mathbb{Z} \cong \operatorname{Mat}_{k, k} R,
$$

where $R$ is a order in $\mathcal{O}_{K\left(\xi_{n}\right)}$ isomorphic to the ring of matrices (not necessarily with unit determinant) with coefficients in $\mathcal{O}_{K}$ commuting with $T_{n}$ And the invertible ones with integer coefficients are

$$
C(T) \cong G L_{k} R \subset G L_{k}\left(\mathcal{O}_{K\left(\xi_{n}\right)}\right) .
$$

The following two propositions give bases for the proof of the vanishing results, namely theorem 0.1 and theorem 0.2 .

Proposition 2.7. Let $A$ be a torsion element of $G L_{m} \mathcal{O}_{K}$. Then $\chi(C(A)) \neq 0$ if and only if the set of eigenvalues of $A$ is inside the set

(a) $\left\{1,-1, i,-i, \xi_{3}, \bar{\xi}_{3}, \xi_{6}, \bar{\xi}_{6}\right\}$, and the multiplicity of 1 and -1 is at most 2 and the multiplicity of the rest of the roots of unity is at most 1 if $K=\mathbb{Q}$;

(b) $\{1,-1, i,-i\}$, and the multiplicities are at most 1 if $K=\mathbb{Q}(i)$;

(c) $\left\{1,-1, \xi_{3}, \bar{\xi}_{3}, \xi_{6}, \bar{\xi}_{6}\right\}$ and the multiplicities are at most 1 if $K=\mathbb{Q}\left(\xi_{3}\right)$;

(d) $\{1,-1\}$, and the multiplicities are at most 1 if $K=\mathbb{Q}(\sqrt{-d}), d \neq 3,4$.

(e) $\chi(C(A))=0$ always when $K \neq \mathbb{Q}, \mathbb{Q}(\sqrt{-d})$.

For $S L_{m} \mathcal{O}_{K}$ we have a similar statement.

Proposition 2.8. Let $A$ be a torsion element of $S L_{m} \mathcal{O}_{K}$. Then $\chi(C(A)) \neq 0$ if and only if the set of eigenvalues of $A$ is inside the set

(a) $\left\{1,-1, i,-i, \xi_{3}, \bar{\xi}_{3}, \xi_{6}, \bar{\xi}_{6}\right\}$, and the multiplicity of 1 is at most 2 , the multiplicity of -1 is 0 or 2 and the multiplicity of the rest of the roots of unity is at most 1 if $K=\mathbb{Q}$;

(b) $\{1,-1, i,-i\}$, and the multiplicities are at most 1 if $K=\mathbb{Q}(i)$;

(c) $\left\{1,-1, \xi_{3}, \bar{\xi}_{3}, \xi_{6}, \bar{\xi}_{6}\right\}$ and the multiplicities are at most 1 if $K=\mathbb{Q}\left(\xi_{3}\right)$; 
(d) $\left\{\xi, \xi^{-1}\right\}$ where $\xi$ is a root of 1 and the dimension of the matrix $A$ is at most 2 , if $K$ is totally real field different from $\mathbb{Q}$.

(e) $\chi(C(A))=0$ always when $K$ is not totally real and different from $\mathbb{Q}(i)$ and $\mathbb{Q}\left(\xi_{3}\right)$.

The following theorems are very useful for computational purposes. They express the homological Euler characteristics as a sum of very few terms. And each of the terms can be easily computed. We use the following notation:

$$
A=\left[A_{11}, \ldots A_{l l}\right]
$$

means that the square block $A_{11}$ through $A_{l l}$ are placed on the block-diagonal of $A$ and the blocks of $A$ outside the block-diagonal are zero blocks. Also, let

$$
R(A)=R\left(f_{1}, \ldots, f_{l}\right)=\prod_{i<j} R\left(f_{i}, f_{j}\right),
$$

where

$$
A=\left[A_{11}, \ldots A_{l l}\right]
$$

and $f_{i}$ is the characteristic polynomial of $A_{i i}$, and $f_{i}$ is a power of an irreducible polynomial. As a consequence of proposition 2.3 and 2.7 we obtain theorems $2.9,2.10,2.11$, and 2.12 .

Theorem 2.9. Let $V$ be a finite dimensional representation of $G L_{m}(\mathbb{Q})$. Then the homological Euler characteristic of $G L_{m} \mathbb{Z}$ with coefficients in $V$ is given by

$$
\chi_{h}\left(G L_{m}(\mathbb{Z}), V\right)=\sum_{A}|R(A)| \chi(C(A)) \operatorname{Tr}\left(A^{-1} \mid V\right),
$$

where the sum is taken over torsion matrices $A$ consisting of blocks $A_{11}, \ldots A_{l l}$ on the block-diagonal and zero blocks off the diagonal. Also the matrices $A_{i i}$ are in the set $\left\{+1,+I_{2},-1,-I_{2}, T_{3}, T_{4}, T_{6}\right\}$, where

$$
T_{3}=\left[\begin{array}{rr}
0 & 1 \\
-1 & -1
\end{array}\right], T_{4}=\left[\begin{array}{rr}
0 & 1 \\
-1 & 0
\end{array}\right], T_{6}=\left[\begin{array}{rr}
0 & -1 \\
1 & 1
\end{array}\right] .
$$

and the characteristic polynomial $f_{i}$ of $A_{i i}$ is a power of an irreducible polynomial, and $f_{i}$ and $f_{j}$ are relatively prime.

Theorem 2.10. Let $V$ be a finite dimensional representation of $G L_{m}(\mathbb{Q}(i))$. Then the homological Euler characteristic of $G L_{m}(\mathbb{Z}[i])$ with coefficients in $V$ is given by

$$
\chi_{h}\left(G L_{m}(\mathbb{Z}[i]), V\right)=\sum_{A}\left|N_{\mathbb{Q}(i) / \mathbb{Q}}(R(A))\right| \chi(C(A)) \operatorname{Tr}\left(A^{-1} \mid V\right),
$$

where the sum is taken over torsion matrices $A$ consisting of blocks $A_{11}, \ldots A_{m m}$ on the block-diagonal and zero blocks off the diagonal. Also the matrices $A_{i i}$ are in the set $\{+1,-1, i,-i\}$ and the characteristic polynomials $f_{i}$ of $A_{i i}$ are relatively prime if $i \neq j$. 
Theorem 2.11. Let $V$ be a finite dimensional representation of $G L_{m}\left(\mathbb{Q}\left(\xi_{3}\right)\right)$. Then the homological Euler characteristic of $G L_{m}\left(\mathbb{Z}\left[\xi_{3}\right]\right)$ with coefficients in $V$ is given by

$$
\chi_{h}\left(G L_{m}\left(\mathbb{Z}\left[\xi_{3}\right]\right), V\right)=\sum_{A}\left|N_{\mathbb{Q}\left(\xi_{3}\right) / \mathbb{Q}}(R(A))\right| \chi(C(A)) \operatorname{Tr}\left(A^{-1} \mid V\right),
$$

where the sum is taken over torsion matrices $A$ consisting of blocks $A_{11}, \ldots A_{m m}$ on the block-diagonal and zero blocks off the diagonal. Also the matrices $A_{i i}$ are in the set $\left\{+1,-1, \xi_{3}, \overline{\xi_{3}}, \xi_{6}, \overline{\xi_{6}}\right\}$ and the characteristic polynomials $f_{i}$ of $A_{i i}$ are relatively prime if $i \neq j$.

Theorem 2.12. Let $P$ be a projective module of rank 2 over the ring of integers $\mathcal{O}_{K}$ in a number field $K=\mathbb{Q}(\sqrt{-d})$ for $d \neq 3,4$. Let $V$ be a finite dimensional representation of $G L_{2}(\mathbb{Q}(\sqrt{-d}))$ Then the homological Euler characteristic of $\operatorname{Aut}(P)$ with coefficients in $V$ is given by

$$
\chi_{h}(\operatorname{Aut}(P), V)=h \cdot \operatorname{Tr}([1,-1] \mid V),
$$

where $h$ is the class number of $\mathbb{Q}(\sqrt{-d})$. In particular, if $P$ is free we obtain the homological Euler characteristic of $G L_{2}\left(\mathcal{O}_{\mathbb{Q}(\sqrt{-d})}\right)$ with coefficients in any representation of $G L_{2}(K)$.

\section{Computation of homological Euler characteristics}

With our method we obtain the following known results.

Theorem 3.1. Let $S^{n} V_{2}$ be the $n$-th symmetric power of the standard representation of $G L_{2}(\mathbb{Q})$. Then

$$
\begin{aligned}
& \chi_{h}\left(G L_{2} \mathbb{Z}, S^{12 n+k} V_{2}\right)=\left\{\begin{array}{cl}
-n+1 & k=0 \\
-n & k=2 \\
-n & k=4 \\
-n & k=6 \\
-n & k=8 \\
-n-1 & k=10 \\
0 & k=\text { odd },
\end{array} \quad \chi_{h}\left(G L_{2} \mathbb{Z}, S^{12 n+k} V_{2} \otimes \text { det }\right)=\right. \\
& \left\{\begin{array}{cl}
-n & k=0 \\
-n-1 & k=2 \\
-n-1 & k=4 \\
-n-1 & k=6 \\
-n-2 & k=8 \\
0 & k=10
\end{array}\right. \\
&
\end{aligned}
$$

Using theorems 2.9, 2.10 and 2.11 we obtain the following four statements.

Theorem 3.2. Let $V_{3}$ and $V_{4}$ be the standart 3 and 4 dimensional representations of $G L_{3} \mathbb{Z}$ and $G L_{4} \mathbb{Z}$ over the rational numbers. Then

$$
\chi_{h}\left(G L_{4} \mathbb{Z}, S^{n} V_{4}\right)=\chi_{h}\left(G L_{3} \mathbb{Z}, S^{n} V_{3}\right)=\chi_{h}\left(G L_{2} \mathbb{Z}, S^{n} V_{2}\right),
$$




$$
\chi_{h}\left(G L_{4} \mathbb{Z}, S^{n} V_{4} \otimes \text { det }\right)=\chi_{h}\left(G L_{2} \mathbb{Z}, S^{n+2} V_{2} \otimes \text { det }\right)
$$

\section{Theorem 3.3.}

$$
\begin{aligned}
\chi_{h}\left(G L_{5} \mathbb{Z}, \mathbb{Q}\right)= & 0, \chi_{h}\left(G L_{6} \mathbb{Z}, \mathbb{Q}\right)=1, \chi_{h}\left(G L_{7} \mathbb{Z}, \mathbb{Q}\right)=-1, \\
& \chi_{h}\left(G L_{8} \mathbb{Z}, \mathbb{Q}\right)=1, \chi_{h}\left(G L_{9} \mathbb{Z}, \mathbb{Q}\right)=-1, \chi_{h}\left(G L_{10} \mathbb{Z}, \mathbb{Q}\right)=1 .
\end{aligned}
$$

Theorem 3.4. The homological Euler characteristic of $G L_{m}(\mathbb{Z}[i])$ with coefficients the symmetric powers of the standard representation are given by

$$
\chi_{h}\left(G L_{m}(\mathbb{Z}[i]), S^{n} V_{m}\right)=\left\{\begin{array}{ll}
1 & n \equiv 0 \bmod 4, \\
0 & \text { otherwise. }
\end{array} \quad \text { for } m=2,3,4,\right.
$$

and constantly zero for $m>4$.

Theorem 3.5. The homological Euler characteristic of $G L_{m}\left(\mathbb{Z}\left[\xi_{3}\right]\right)$, where $\xi_{3}$ is a non-trivial third root of 1 , with coefficients the symmetric powers of the standard representation are given by

$$
\chi_{h}\left(G L_{m}\left(\mathbb{Z}\left[\xi_{3}\right]\right), S^{n} V_{m}\right)=\left\{\begin{array}{ll}
1 & n \equiv 0 \bmod 6, \\
0 & \text { otherwise. }
\end{array} \quad \text { for } m=2,3,4,5,6,\right.
$$

and constantly zero for $m>6$.

Lemma 3.6. Let $G$ be a group and $\Gamma$ be a subgroup. Given an element $A \in \Gamma$, the set of elements in which are conjugate to $A$ in $G$ but not in $\Gamma$ is parameterized by the elements of the double quotient

$$
\Gamma \backslash N_{G}^{\Gamma}(A) / C_{G}(A),
$$

where $N_{G}^{\Gamma}(A)=\left\{X \in G: X A X^{-1} \in \Gamma\right\}$ and $C_{G}(A)$ is the centralizer of $A$ inside the group $G$.

Let $\Gamma_{1}(m, N)$ be the subgroup of $G L_{m}(\mathbb{Z})$ that fixes the covector $[0, \ldots, 0,1]$ modulo $N$.

Lemma 3.7. Let $A \in \Gamma_{1}(m, N)$ be a torsion element with $\chi(C(A)) \neq 0$, let $N$ be relatively prime with 2 and 3 and let $f$ be the characteristic polynomial of $A$. Then +1 is a root of $f$ and it has multiplicity 1 or 2 .

(a) If the root +1 has multiplicity 1 then the set

$$
\Gamma_{1}(m, N) \backslash N_{C L_{m} \mathbb{Z}}^{\Gamma_{1}(m, N)}(A) / C_{G L_{m} \mathbb{Z}}(A)
$$

has $\frac{1}{2} \varphi(N)$ elements.

(b) If the root +1 has multiplicity 2 then the set

$$
\Gamma_{1}(m, N) \backslash N_{C L_{m} \mathbb{Z}}^{\Gamma_{1}(m, N)}(A) / C_{G L_{m} \mathbb{Z}}(A)
$$

has one element. 
The proof is long and it can be found in [Ho].

Theorem 3.8. Let $V$ be a representation of $G L_{m} \mathbb{Z}$, and let $N$ be an integer relatively prime to 2 and 3 . Then

$$
\begin{aligned}
\chi_{h}\left(\Gamma_{1}(m, N), V\right)= & \varphi(N) \sum_{A=\left[A_{1}, 1\right]} R(A) \chi\left(C_{G L_{m-1} \mathbb{Z}}\left(A_{1}\right)\right) \operatorname{Tr}\left(A^{-1} \mid V\right)+ \\
& +\varphi_{2}(N) \sum_{A=\left[A_{2}, I_{2}\right]} R(A) \chi\left(C_{G L_{m-2} \mathbb{Z}}\left(A_{2}\right)\right) \operatorname{Tr}\left(A^{-1} \mid V\right),
\end{aligned}
$$

where $A_{1}$ and $A_{2}$ are block-diagonal matrices with zero blocks off the diagonal, and with block on the diagonal $A_{i i}$ varying through

$$
\left\{-1,-I_{2}, T_{3}, T_{4}, T_{6}\right\}
$$

and $A_{i i}$ and $A_{j j}$ have no common eigenvalues. We set

$$
T_{3}=\left[\begin{array}{rr}
0 & 1 \\
-1 & -1
\end{array}\right], T_{4}=\left[\begin{array}{rr}
0 & 1 \\
-1 & 0
\end{array}\right], T_{6}=\left[\begin{array}{rr}
0 & -1 \\
1 & 1
\end{array}\right] .
$$

Also $\varphi(N)$ is the Euler function of $N$, and $\varphi_{2}(N)$ is the arithmetic function generated by

$$
\varphi_{2}\left(p^{n}\right)=p^{2 k}\left(1-\frac{1}{p^{2}}\right) .
$$

Corollary 3.9. For $N$ not divisible by 2 and 3 the homological Euler characteristics of $\Gamma_{1}(m, N)$ with coefficients the symmetric powers of $V_{m}$ are given by

$$
\begin{aligned}
& \quad \chi_{h}\left(\Gamma_{1}(2, N), S^{2 n+k} V_{2}\right)= \begin{cases}-\frac{1}{24} \varphi_{2}(N)(2 n+1)+\frac{1}{2} \varphi(N) & k=0 \\
-\frac{1}{24} \varphi_{2}(N)(2 n+2) & k=1\end{cases} \\
& \begin{array}{l}
\chi_{h}\left(\Gamma_{1}(3, N), \mathbb{Q}\right)=-\frac{1}{12} \varphi_{2}(N)+\frac{1}{2} \varphi(N) . \\
\chi_{h}\left(\Gamma_{1}(4, N), \mathbb{Q}\right)=\varphi(N),
\end{array} \\
& \text { where } \varphi(N) \text { is the multiplicative Euler function generated by } \varphi\left(p^{n}\right)=p^{n}\left(1-\frac{1}{p}\right) \\
& \text { and } \varphi_{2}(N) \text { is the multiplicative function generated by } \varphi_{2}\left(p^{n}\right)=p^{2 n}\left(1-\frac{1}{p^{2}}\right) .
\end{aligned}
$$

For the rings $\mathbb{Z}[i]$ and $\mathbb{Z}\left[\xi_{3}\right]$ we can similarly define $\Gamma_{1}(m, \mathfrak{a})$ for an ideal $\mathfrak{a}$ to be the stabilizer of $[0, \ldots, 0,1]$ in $G L_{m}$ over the ring, modulo a. For these arithmetic groups we have similar formulas to lemma 3.7 part(a). Consequently, we obtain the homological Euler characteristics given in theorem 0.5.

Proof. (of theorem 0.6) We use Brown's formula. For $S L_{2}\left(\mathcal{O}_{K}\right)$ for totally real number fields $K$, the torsion elements are either $\pm I_{2}$ or a matrix $A$ with irreducible polynomial over $K$ with roots $\xi$ and $\xi^{-1}$ primitive $n$-root of 1 . The matrices $I_{2}$ and $-I_{2}$ give two copies of $\zeta_{k}(-1)$. For the matrices $A$, let $I$ be the corresponding ideal by proposition 1.3. And let $R_{I} \subset \mathcal{O}_{K}[\xi]$ be isomorphic to the ring of matrices that commute with $A$. Then the centralizer of $A$ in $S L_{2}\left(\mathcal{O}_{K}\right)$ correspond to the torsion elements in $R_{I}$. Also,

$$
S L_{2}\left(\mathcal{O}_{K}\right) \backslash N_{G L_{2} \mathcal{O}_{K}}^{S L_{2} \mathcal{O}_{K}}(A) / C_{G L_{2} \mathcal{O}_{K}}(A)=\mathcal{O}_{K}^{\times} / N_{K(\xi) / K}\left(R_{I}^{\times}\right) .
$$

This proves the theorem. 
Note the units and the torsion elements in the orders $R_{I}$ give information about the torsion part of $\zeta_{K}(-1)$.

Example 3.10. Consider $K=\mathbb{Q}(\sqrt{5})$. Then all the quadratic extension of $K$ be $n$-th root of 1 lead to field with trivial class number. One can conclude that by decomposing $\zeta_{K(\xi)}(s)$ as a product of Riemann zeta fuction and $L$-functions, and using the residue formula at $s=1$. From theorem 0.5 we obtain

$$
\chi_{h}\left(S L_{2}(\mathbb{Z}[(1+\sqrt{5}) / 2), \mathbb{Q})=2 \cdot \zeta_{\mathbb{Q}(\sqrt{5})}(-1)+3+\frac{14}{15}\right.
$$

Since the homological Euler characteristic is an integer, call it $N$, we obtain that

$$
\zeta_{\mathbb{Q}(\sqrt{5})}(-1)=-\frac{14}{30}+\frac{N}{2} .
$$

In fact, $\zeta_{\mathbb{Q}(\sqrt{5})}(-1)=1 / 30$ see $[\mathrm{I}]$ theorem 1 . Thus, $\chi_{h}\left(S L_{2}(\mathbb{Z}[(1+\sqrt{5}) / 2), \mathbb{Q})=\right.$ 4 .

\section{Generalization of Brown's formula}

Theorem 4.1. The homological Euler characteristic of $\Gamma$ with coefficients in $V$ is given by

$$
\chi_{h}(\Gamma, V)=\sum \chi(C(A)) \cdot \operatorname{Tr}\left(A^{-1} \mid V\right),
$$

where the sum is taken over all torsion elements of $\Gamma$ counted up to conjugation, $C(A))$ is the centralizer of $A$ inside $\Gamma$ and $\operatorname{Tr}\left(A^{-1} \mid V\right)$ is the trace of the action of $A^{-1}$ on the finite dimensional vector space $V$.

Proof. This proof follows more closely the paper of Brown [B2]. And it turn out that the formula we need is a consequence of a more general formula of Brown for a finite length chain $C^{\bullet}$ of $\mathbb{Q} \Gamma$-modules that admit generalized Euler characteristic.

For an endomorphism of a finitely generated projective $\mathbb{Q} \Gamma$-module $P$ one can define its trace with values in $\mathbb{Q} \Gamma /[\mathbb{Q} \Gamma, \mathbb{Q} \Gamma]$. Then $E(\Gamma, P)=\operatorname{Tr}_{\mathbb{Q} \Gamma}\left(i d_{P}\right)$. If a $\mathbb{Q} \Gamma$-module $V$ admits a finite length projective resolution $P_{\bullet}$ then $E(\Gamma, V)=$ $\sum_{i}(-1)^{i} E\left(\Gamma, P_{i}\right)$. For more information on this trace see [B1], [B2], [Ba]. For the relation to the homological Euler chracteristic see [Ch]. Define

$$
E\left(\Gamma, C^{\bullet}\right)=\sum_{i}(-1)^{i} E\left(\Gamma, C^{i}\right)
$$

to be the generalized Euler characteristic Let

$$
E\left(\Gamma, C^{\bullet}\right)=\sum_{(A)} c_{(A)} \cdot(A)
$$

Define

$$
e\left(\Gamma, C^{\bullet}\right)=c_{(I)},
$$

where $I$ is the identity element in $\Gamma$. We have that

$$
e(\Gamma, \mathbb{Q})=\chi(\Gamma) .
$$


The general formula that Brown obtains is that $c_{(A)}$ coincides with the coefficient next to $(I)$ in $\operatorname{Tr}_{\mathbb{Q} \Gamma}\left(M_{A}\right)$, where $M_{A}:\left(C^{\bullet}\right)^{A} \rightarrow\left(C^{\bullet}\right)^{A}$ is multiplication by $A$. Now, let $X$ be a contractable CW complex on which $\Gamma$ act properly and discontinuously. One can assume also that a cell is mapped to itself by an element of $\Gamma$ then the entire cell is fixed by that element pointwise. One can achieve that by subdivision of the cells. Then

$$
E(\Gamma, V)=E\left(\Gamma, C^{\bullet}(X, V)\right),
$$

where $C^{\bullet}(X, V)$ is the cochain complex of $X$ with coefficients in $V$. Let $f \in$ $C^{i}(X, V)$ and $A \in \Gamma$. let also $\sigma$ be an $i$-th cell and $v \in V$ so that $f(\sigma)=v$. Then $A$ acts on $f$ by $(A \cdot f)(A \sigma)=A \cdot v$. Since we want to find the trace we consider the fixed cells under the action of $A$. Consider a the following basis for $C^{i}(X, V)$. Let $v_{1}, \ldots, v_{n}$ be a basis for $V$. Let $f_{\sigma, v_{i}}$ be the function that sends the cell $\sigma$ to the vector $v_{i}$, and sends all other cell to zero. Consider the contribution of $f_{\sigma, v_{i}}$ to the trace. We have that $A \cdot f_{\sigma, v_{i}}$ maps $\sigma$ to $a_{i i} \cdot v_{i}$, where $a_{i i}$ is a constant. But $A \cdot f_{\sigma, v_{i}}$ maps $A \sigma$ to $A v_{i}$. Thus, we have a contribution to the trace if

$$
A \sigma=\sigma .
$$

Consider the space $X^{A}$ of cells which are fixed under the action of $A$. On $X^{A}$ the centralizer $C(A)$ acts properly and discontinuously. Also $X^{A}$ is contractable. Thus

We have

$$
c_{(A)}=e\left(C(A), C\left(X^{A}, V\right)\right) .
$$

$$
(A \cdot f)(\sigma)=A^{-1}(f(A \sigma))=A^{-1}(f(\sigma)) .
$$

When we take the trace the last equation leads to $\operatorname{Tr}\left(A^{-1} \mid V\right)$. Thus, we obtain

$$
c_{(A)}=e\left(C(A), C^{\bullet}\left(X^{A}\right)\right) \cdot \operatorname{Tr}\left(A^{-1} \mid V\right)=\chi(C(A)) \cdot \operatorname{Tr}\left(A^{-1} \mid V\right) .
$$

\section{Acknowledgements}

I would like to thank most of all my advisor professor Alexander Goncharov for constant encouragement for my work and for giving me a series of problems that lead to this thesis. I would like to thank professors Michael Rosen, Bruno Harris, and Stephen Lichtenbaum for the discussions we had. I would like to thank professor Thomas Goodwillie for pointing out a mistake which I had to correct, and professor Jean-Pierre Serre for a useful conversation. And also I would like to thank Amir Jafary for the many useful conversations we had that contributed to this paper.

\section{References}

[Ba] H. Bass, Euler Characteristic and Characters of Discrete Groups, Inventiones math. 35 (1976), 155-196.

[B1] K. Brown, Cohomology of Groups, Graduate Text in Mathematics, Springer-Verlag: New York, 1982.

[B2] Complete Euler characteristics and fixed-point theory, J. Pure Appl. Algebra 24 (1982), 103-121. 
[Ch] I. Chiswell, Euler characteristics of groups, Math. Z. 147, (197) 1-11.

[G1] A. Goncharov, The dihedral Lie algebras and the Galois symmetries of $\pi_{1}\left(P^{1}-\right.$ $\left.\{0, \infty\} \cup \mu_{n}\right)$, Duke Math. J. 110 (2001), 397-487.

[H] G. Harder, A Gauss-Bonnet formula for discrete arithmetically defined groups, Ann. Sci. École Norm. Sup.(4) 4 (1971), 409-455.

[Ho] I. Horozov, Euler characteristics of arithmetic groups, http://xxx.lanl.gov/, No: math.GR/0311117, p. 94.

[I] K. Iwasawa, Lecutures on p-adic L-functions, Annals of Math Studies 74 Princeton Univ. Press: Princeton, N.J., 1972.

[M] J. Milnor, Introduction to algebraic K-theory Ann. of Math Studies 72, Princeton University Press, Princeton, 1971.

[S] Serre, J.-P.: Cohomologie des groupes discretes, Ann. of Math. Studies 70 (1971), 77-169.

[T] J. Tate, Symbols in Arithmetic, Proc. of Int Congress of Math. at Nice (1970).

[W] L. Washington, Introduction to cyclotomic fields Graduate Text in Mathematics, Springer-Verlag: New York, 1982.

Max Planck Institut für Mathematik, Vivatsgasse 7, Bonn 53111, Germany

E-mail address: horozov@mpim-bonn.mpg.de 